\title{
Avaliação hemogasométrica em cães submetidos à pneumonectomia esquerda ${ }^{1}$
}

\author{
Daniella H. Binoki ${ }^{2}$, Fabio B. Jatene ${ }^{3}$, Denise T. Fantoni ${ }^{4}$, Angelo J. Stopiglia ${ }^{4 *}$, André \\ L.S. Santos ${ }^{5}$, Rodrigo R. Freitas ${ }^{2}$, Edson A. Simões ${ }^{5}$, Eduardo T. Irino ${ }^{5}$, Rosangela \\ Monteiro $^{3}$ e Renato Lofiego ${ }^{2}$
}

\begin{abstract}
Binoki D.H., Jatene F.B., Fantoni D.T., Stopiglia A.J., Santos A.L.S., Freitas R.R., Simões E.A., Irino E.T., Monteiro R. \& Lofiego R. 2007. [Hemogasometric evaluation in dogs submitted to left pneumonectomy.] Avaliação hemogasométrica em cães submetidos à pneumonectomia esquerda. Pesquisa Veterinária Brasileira 27(8):345-351. Departamento de Cirurgia, Faculdade de Medicina Veterinária e Zootecnia, Universidade de São Paulo, São Paulo, SP 05508-270, Brazil. E-mail: stopa@usp.br

It is well known that different diseases of infectious, traumatic or neoplasic origin can occur in the lungs of dogs, and pneumonectomy technique may be an option for the treatment of some of these diseases. The objective was to evaluate hemogasometric parameters, oxygen partial pressure $\left(\mathrm{PaO}_{2}\right)$, carbon dioxide partial pressure $\left(\mathrm{PaCO}_{2}\right)$, hydrogen ion concentration $(\mathrm{pH})$ and bicarbonate ion concentration on the pre- and postoperative moments of the left pneumonectomy. Eighteen adult mongrel dogs, males and females, were randomly distributed into two groups with 9 dogs each. In Group A, the left bronchial stump of the dogs was sutured manually with polypropylene 5-0, and, in Group $B$, the left bronchial stump of the dogs was sutured mechanically with a surgical stapler. The data were collected at 6 moments: Before the pre-anesthetic administration (T0), one hour after the extubation (T1EXT), 48 hours after the surgery (T48h), 7 days after the surgery (T7d), 15 days after the surgery (T15d), and 36 days after the surgery (T36d). The results were statistically analyzed. $\mathrm{PaO}_{2}$ values of Group A on T1EXT (67.00 \pm 11.31 ) were significantly lower in relation to T0 $(99.4 \pm 18.34)$, a fact that did not happen in Group B: T1EXT $(87.00 \pm 8.35)$ in regard to T0 $(87.00 \pm 7.55)$. There was no difference on $\mathrm{pH}$ values in dogs of Group A, but in Group B was observed a decrease on T1EXT (7.3644 \pm 0.0353$)$ in relation to T0 $(7.4189 \pm 0.0136)$, although the animals did not develop acidosis. It was concluded that dogs submitted to left pneumonectomy (sutured manually or sutured mechanically of left bronchial) did not show immediate and mediate acid-basic instability during the study.
\end{abstract}

INDEX TERMS: Pneumonectomy, manual suture, mechanic suture, hemogasometric, dogs.

\footnotetext{
${ }^{1}$ Recebido em 10 de janeiro de 2007.

Aceito para publicação em 6 de junho de 2007.

${ }^{2}$ Médico Veterinário autônomo, Rua Ibituruna 283, apto 103, Saúde, São Paulo, SP 04302-051, Brasil.

${ }^{3}$ Serviço de Cirurgia Torácica, Departamento de Cardiopneumologia, Faculdade de Medicina, Universidade de São Paulo (USP), Av. Dr. Enéas de Carvalho Aguiar 44, 5ํandar, São Paulo, SP 05403-900, Brasil.

${ }^{4}$ Laboratório de Cirurgia Cardiotorácica (LCCT), Departamento de Cirurgia, Faculdade de Medicina Veterinária e Zootecnia (FMVZ), USP, Av. Prof. Dr. Orlando Marques de Paiva 87, São Paulo, SP 05508-270, Brasil. *Autor para correspondência: stopa@usp.br

${ }^{5}$ Pós-Graduando, LCCT, Departamento de Cirurgia, FMVZ, USP. Endereço: Av. Francisco Leme 353, Parque Fongaro, São Paulo, SP 04257-000, Brasil.
}

RESUMO.- Moléstias de natureza infecciosa, traumática ou neoplásica acometem o pulmão dos cães, constituindo-se a pneumonectomia como possibilidade de tratamento para algumas dessas afecções. Assim, diante da escassez de dados encontrados na literatura, objetivou-se avaliar e comparar parâmetros hemogasométricos, pressão parcial de oxigênio $\left(\mathrm{PaO}_{2}\right)$, de dióxido de carbono $\left(\mathrm{PaCO}_{2}\right)$, concentração do íon hidrogênio $(\mathrm{pH})$ e do íon bicarbonato [HCO3-] no sangue arterial de cães adultos, antes e após pneumonectomia esquerda. Foram estudados 18 cães, machos e fêmeas de idades variadas, pesando entre 15 e $20 \mathrm{~kg}$, distribuídos aleatoriamente em dois grupos de 9 animais cada. No Grupo A, o coto brôn- 
quico esquerdo dos cães foi suturado manualmente com fio de polipropileno 5-0, e no Grupo B, o coto brônquico esquerdo foi suturado mecanicamente com grampeador cirúrgico. Os dados foram colhidos em 6 momentos: antes da administração da medicação pré-anestésica (T0), 1 hora após a extubação do animal (T1EXT), 48 horas após a intervenção cirúrgica (T48h), 17 (T7d), 15 (T15d) e 36 dias (T36d) após intervenção cirúrgica. Em seguida, foi realizada a análise estatística (teste de normalidade de Anderson-Darling, Wilcox e teste $\mathrm{U}$ de Man-Whitney). Os valores de $\mathrm{PaO}_{2}$ do Grupo A no T1EXT $(67,00 \pm 11,31)$ mostraram-se significativamente inferiores em relação ao T0 $(99,44 \pm 18,34)$, fato este que não ocorreu no Grupo B: T1EXT $(87,00 \pm 8,35)$ em relação ao T0 $(87,00 \pm 7,55)$. Não houve diferença do $\mathrm{pH}$ entre os momentos nos cães do grupo A, porém no grupo B observou-se uma diminuição em T1EXT $(7,3644 \pm 0,0353)$ em relação ao T0 $(7,4189 \pm 0,0136)$, sem que os animais tenham desenvolvido acidose. Apesar dessas alterações, concluiu-se que os cães submetidos à pneumonectomia esquerda (sutura manual ou mecânica do coto brônquico esquerdo) não apresentaram tendência a desenvolver desequilíbrio ácido-básico no período em que foram avaliados.

TERMOS DE INDEXAÇÃO: Pneumonectomia, sutura manual, sutura mecânica, hemogasometria, cães.

\section{INTRODUÇÃO}

Assim como no homem, os pulmões dos cães podem ser afetados por enfermidades, sejam elas congênitas ou adquiridas, degenerativas, inflamatórias, infecciosas ou não infecciosas, parasitárias, traumáticas e neoplásicas. Na dependência da gravidade e do grau de comprometimento tecidual, em alguns casos, há indicação da excisão da região afetada através de lobectomia ou pneumonectomia (Hawkins 2006).

A pneumonectomia é procedimento operatório satisfatoriamente aplicado em Medicina Humana para o tratamento de diversas doenças pulmonares (Goire et al. 1993). Tal procedimento também pode ser aplicado com sucesso para o tratamento de enfermidades em pequenos animais. Porém, segundo Matsumoto et al. (2004), a pneumonectomia não é indicada em alguns pacientes que apresentam disfunção pulmonar, visto que a intervenção cirúrgica geralmente conduz à falência respiratória.

Em continuidade à linha de estudos sobre cirurgias pulmonares em cães, onde avaliaram-se, na pneumonectomia esquerda, os aspectos anatomopatológicos, radiológicos e broncoscópicos do pulmão, além dos paramétricos do animal (Simões 2003, Simões et al. 2005); os dados histopatológicos do pulmão direito e a avaliação eletrocardiográfica dos animais submetidos à pneumonectomia esquerda (Irino et al. 2004), além de, na pneumonectomia direita, onde estudou-se, histologicamente, o pulmão esquerdo, bem como as alterações eletrocardiográficas dos cães (Irino 2006), objetivou-se, neste trabalho, avaliar, especificamente, as possíveis alterações na tensão de oxigênio arterial $\left(\mathrm{PaO}_{2}\right)$, tensão de dióxido de carbono arterial $\left(\mathrm{PaCO}_{2}\right)$, concentração de hidrogênio $(\mathrm{pH})$, e concentração de íons bicarbonato [HCO3], nos períodos pré e trans-opera- tórios de cães submetidos à pneumonectomia esquerda, a fim de se determinar a magnitude das alterações hemogasométricas provocadas pela intervenção cirúrgica.

\section{MATERIAL E MÉTODOS}

O experimento foi realizado no Laboratório de Cirurgia Cardiotorácica do Departamento de Cirurgia, Faculdade de Medicina Veterinária e Zootecnia, Universidade de São Paulo. Foram avaliados 18 cães sem raça definida (Canis familiaris), adultos, machos e fêmeas, pesando entre 15 e $20 \mathrm{~kg}$, provenientes de Centros de Controle de Zoonoses localizados na Grande São Paulo. Os animais utilizados eram hígidos e isentos de quaisquer alterações que porventura comprometessem a realização desse estudo.

Os cães foram tratados contra ectoparasitas e endoparasitas com ivermectina a $1 \%(400 \mathrm{mmg} / \mathrm{kg})$, dipropionato de imidocarb $(5 \mathrm{mg} / \mathrm{kg})$ e banhados com amitraz 15 dias antes do procedimento cirúrgico. No período correspondente a 24 horas antes da intervenção cirúrgica, receberam benzilpenicilina benzatina profilática (40000 U.I./kg). Igualmente, os animais foram submetidos a jejum hídrico e alimentar de 6 e 12 horas, respectivamente. Durante o transcorrer do projeto, receberam cuidados necessários de acordo com o Colégio Brasileiro de Experimentação Animal (COBEA), sendo o projeto aprovado pela Comissão de Bioética da FMVZ/USP (Protocolo 133/2002).

Os animais foram distribuídos aleatoriamente em dois grupos: o primeiro formado por nove cães (Grupo A), cujo coto brônquico esquerdo foi suturado manualmente com fio de polipropileno 5-0 e o segundo (Grupo B) composto pelos demais animais, cujo coto brônquico esquerdo foi suturado mecanicamente com grampeador cirúrgico (Grampeador mecânico TA-55 - Ethicon ${ }^{\circledR}$ ).

Tanto os cães do Grupo A como os do Grupo B foram separados aleatoriamente em três subgrupos compostos por três animais: Subgrupos A1 e B1, formados por cães que passaram por 7 dias de experimento; $\mathrm{A} 2$ e $\mathrm{B} 2$, compostos por animais que permaneceram 15 dias em estudo; e A3 e B3, representados pelos cães que foram observados por 36 dias pela experimentação.

\section{Procedimento anestésico}

A medicação pré-anestésica foi realizada com a administração de morfina $(0,2 \mathrm{mg} / \mathrm{kg})$ associada à acepromazina $(0,1 \mathrm{mg} / \mathrm{kg})$ pela via intramuscular. Decorridos 30 minutos, procedeu-se à indução da anestesia com propofol $(5 \mathrm{mg} / \mathrm{kg})$ pela via intravenosa e, quando atingido o plano anestésico adequado, os cães foram intubados com sonda endotraqueal seletiva (Bronco-Cath, Mallinckrodt), guiada por broncoscópio (Broncoscópio BF-P-20D, Olympus). A manutenção da anestesia foi efetuada com o sevofluorano a 3,75\% empregando-se, para tanto, analisador de gases anestésicos (Tracer 5 , Intermed), com o objetivo de assegurar o $2^{\circ}$ plano de anestesia do $3^{\circ}$ estágio de Guedel. Empregaram-se circuito circular de anestesia e fração inspirada de oxigênio de $80 \%\left(\mathrm{FiO}_{2}-0,8\right)$ (Shogun Evolution 2700, Takaoka). A ventilação dos cães foi controlada mecanicamente no modo volume controlado, com volume corrente (VC) inicial de $10 \mathrm{ml} /$ $\mathrm{kg}$ e frequiência respiratória ajustada para a manutenção do dióxido de carbono arterial entre 35 e $45 \mathrm{mmHg}$. Instantes antes do início da intervenção cirúrgica, administrou-se fentanil ( $5 \mu \mathrm{g} / \mathrm{kg}$ IV). Introduziuse percutaneamente cateter $22 \mathrm{G}$ na artéria metatarsiana que foi acoplado a transdutor de pressão arterial do monitor de parâmetros vitais (HPM 1205A/A30, Hewellet Packard), assegurando a mensuração contínua da pressão arterial sistólica, diastólica e média, durante o ato operatório e no pós-operatório imediato.

O bloqueio neuromuscular foi obtido com a utilização de rocurônio $(0,6 \mathrm{mg} / \mathrm{kg}$, IV), e a sua reversão com neostigmine $(0,08 \mathrm{mg} /$ $\mathrm{kg}$, IV) associado a sulfato de atropina $(0,04 \mathrm{mg} / \mathrm{kg}$, IV), ao término 
do procedimento cirúrgico e com o animal apresentando VC adequado para a reversão e desmame da ventilação controlada. Durante o procedimento anestésico, foi realizada a infusão contínua de Ringer com lactato na velocidade de $10 \mathrm{ml} / \mathrm{kg} / \mathrm{hora}$ e, quando necessário, foram empregados fármacos vasoativos para adequação da pressão arterial. Ao final da anestesia, todos os animais receberam morfina $(0,5 \mathrm{mg} / \mathrm{kg}, \mathrm{SC})$, e cetoprofeno na dose de $2 \mathrm{mg} / \mathrm{kg}$ IV associada a dipirona $(25 \mathrm{mg} / \mathrm{kg})$.

\section{Procedimento cirúrgico}

Os cães foram posicionados em decúbito lateral direito e submetidos à ressecção pulmonar esquerda. $\mathrm{O}$ acesso cirúrgico foi realizado por toracotomia lateral esquerda, no quinto espaço intercostal.

A musculatura da parede torácica foi incisada até o plano dos arcos costais, para possibilitar o acesso à cavidade torácica por meio da secção de músculos intercostais e pleura parietal. Após o afastamento lateral dos pulmões, foram identificadas as estruturas do hilo pulmonar. Com o tronco pulmonar identificado, o ramo esquerdo da artéria pulmonar foi duplamente ligado, proximal e distalmente, com fio de náilon 2-0. Após a secção da artéria pulmonar esquerda, o coto proximal foi submetido à sutura de reforço com chuleio vaivém com fio de polipropileno $5-0$. As veias pulmonares foram ligadas duplamente com fio de náilon 2-0 e, em seguida, seccionadas. $\mathrm{O}$ brônquio foi dissecado e uma pinça atraumática foi colocada proximal ao local da secção. A seguir, o brônquio principal esquerdo foi seccionado e o pulmão esquerdo ressecado. $O$ coto proximal remanescente foi tratado segundo o descrito para os Grupos A e B. O grampeador cirúrgico foi utilizado para a secção do brônquio principal esquerdo nos cães do Grupo B.

\section{Acompanhamento do animal no período pós-operatório}

Nas primeiras 24 horas após o ato operatório os cães foram monitorados pela equipe envolvida no experimento, recebendo todos os cuidados pós-operatórios necessários: analgesia, conforto térmico adequado e curativos da ferida cirúrgica, visando a promover a melhor assistência pós-operatória aos animais.

A analgesia foi realizada com a utilização do antiinflamatório cetoprofeno $(2 \mathrm{mg} / \mathrm{kg})$ a cada 24 horas, morfina $(0,5 \mathrm{mg} / \mathrm{kg}, \mathrm{SC})$ e a dipirona $(25 \mathrm{mg} / \mathrm{kg}$, IV), a cada 4 e 8 horas, respectivamente, nas primeiras 48 horas após a pneumonectomia esquerda. 0 cetoprofeno ainda foi administrado ao longo de sete dias.

Para avaliação da dor foi utilizado o protocolo proposto por Thompson \& Johnson (1991) e, no momento da avaliação clínica, os animais receberam nota, variando de 0 a 10 (Quadro 1). Os animais que receberam nota igual ou superior a 5 foram submetidos ao protocolo analgésico com doses adicionais de morfina $(0,1 \mathrm{mg} / \mathrm{kg})$. A aplicação foi repetida toda vez que apresentaram o escore citado.

Durante a avaliação clínica dos animais, foram obtidos dados referentes aos batimentos cardíacos e de movimentos respiratórios com a finalidade de correlacioná-los aos dados avaliados no trabalho.

\section{Avaliação dos parâmetros hemogasométricos}

Foram colhidas anaerobicamente amostras do sangue arterial obtidas por punção da artéria femoral com agulhas e seringas de plástico estéreis e descartáveis, umedecidas interiormente com heparina sódica. Uma vez coletado cerca $0,5 \mathrm{ml}$ de sangue, realizouse gentilmente a homogenização do mesmo com heparina sódica e as extremidades das agulhas foram vedadas com "cubo de borracha".

As amostras foram imediatamente processadas em analisador de gases sanguíneos (ABL-5, Radiometer, Dinamarca), depois de realizado o ajuste da temperatura corpórea do animal no analisador, para obtenção dos seguintes valores: tensão de oxigênio arterial em
Quadro 1. Protocolo para avaliação da dor em cães (Thompson \& Johnson 1991)

\begin{tabular}{llc}
\hline Observação & \multicolumn{1}{c}{ Critério } & Pontos \\
\hline Frequiência & Até 10\% acima do valor pré-operatório & 0 \\
cardíaca & 10-30\% acima do valor pré-operatório & 1 \\
& 30-50\% acima do valor pré-operatório & 2 \\
\multirow{3}{*}{ Ganido } & Mais de 50\% acima do valor pré-operatório & 3 \\
& Sem gemido & 0 \\
& Ganido que corresponde a chamado ou afago & 1 \\
Movimentação & Ganido que não corresponde a chamado ou afago & 2 \\
& Sem movimentação & 0 \\
Agitação & Freqüentemente mudando de posição & 1 \\
& Movimentação freqüiente & 2 \\
& Calmo & 0 \\
& Levemente agitado & 1 \\
Total & Moderadamente agitado & 2 \\
& Histérico & 3 \\
& & 0 a 10
\end{tabular}

$\mathrm{mmHg}\left(\mathrm{PaO}_{2}\right)$; tensão de dióxido de carbono arterial em $\mathrm{mmHg}$ $\left(\mathrm{PaCO}_{2}\right)$; concentração do íon hidrogênio $(\mathrm{pH})$; e concentração do íon bicarbonato, $\mathrm{mEq} / \mathrm{L}\left[\mathrm{HCO}_{3}^{-}\right]$.

Os parâmetros referentes à hemogasometria foram avaliados nos seguintes momentos: antes da administração da medicação préanestésica (T0), 1 hora após a extubação do animal (T1EXT), 48 horas após a intervenção cirúrgica (T48h), 7 (T7d), 15 (T15d) e 36 dias após a operação (T36d).

\section{Análise estatística}

Inicialmente, os valores obtidos da $\mathrm{PaO}_{2}, \mathrm{PaCO}_{2}, \mathrm{pH}$ e $\left[\mathrm{HCO}_{3}^{-}\right]$ dos Grupos A e B nos diversos momentos passaram pelo Teste de Normalidade de Anderson-Darling com grau de significância de 5\%. Para as distribuições obtidas, optou-se pela análise não paramétrica de Wilcoxon de amostras pareadas $(\mathrm{p}=5 \%)$. A avaliação comparativa entre o Grupo A e o Grupo B foi efetuada com o Teste U de ManWhitney para amostras independentes $(\mathrm{p}=5 \%)$. Os cálculos que resultaram em $\mathrm{p}<0,05$, sob intervalo de confiança de $95 \%$, indicaram diferenças significativas entre os avaliados.

\section{RESULTADOS}

Tanto os procedimentos anestésicos como os cirúrgicos demonstraram-se satisfatórios para os 18 cães avaliados, não sendo observadas complicações nos períodos pré, trans e pósoperatórios. Todos os animais apresentaram recuperação completa, não ocorrendo nenhuma morte. Os resultados da $\mathrm{PaO}_{2}$, $\mathrm{PaCO}_{2}, \mathrm{pH}$ e $\left[\mathrm{HCO}_{3}^{-}\right]$no sangue arterial dos animais avaliados estão expostos nos Quadros 2 a 9. Os valores obtidos para batimentos cardíacos e movimentos respiratórios, nos animais dos Grupos A e B, bem como a média e desvio padrão, nos diferentes momentos de coleta de sangue arterial para análise hemogasométrica, estão discriminados nos Quadros 10 a13.

\section{$\mathrm{PaO}_{2}$ (Tensão de oxigênio arterial, $\mathrm{mmHg}$ )}

Pelos resultados obtidos da $\mathrm{PaO}_{2}$ dos Subgrupos A1, A2 e A3, pôde-se constatar que apenas os valores em T1EXT $(p=0,011)$, T48h $(p=0,025)$ e $T 15 d(p=0,043)$ apresentaram resultados estatisticamente inferiores, quando comparados aos valores de T0. Entretanto, apenas os valores obtidos 1 hora após a extubação encontraram-se abaixo dos valores 
Quadro 2. Valores da $\mathrm{PaO}_{2}$ (mmHg) obtidos nos diferentes tempos de avaliação emomentos nos animais submetidos à sutura manual do coto brônquico (Grupo A)

\begin{tabular}{cccccccc}
\hline Subgrupo & Cão & T0 & T1EXT $^{\mathrm{a}}$ & T48h $^{\mathrm{a}}$ & T7d & T15d & T36d \\
\hline 1 & 96 & 52 & 84 & 99 & - & - & \\
$\mathrm{A} 1$ & 2 & 80 & 62 & 80 & 90 & - & - \\
3 & 99 & 59 & 97 & 97 & - & - & \\
4 & 97 & 58 & 71 & 92 & 89 & - & \\
$\mathrm{A} 2$ & 5 & 107 & 77 & 93 & 76 & 82 & - \\
6 & 94 & 83 & 85 & 90 & 87 & - & \\
7 & 143 & 66 & 79 & 140 & 77 & 84 & \\
$\mathrm{~A} 3$ & 8 & 97 & 63 & 75 & 94 & 97 & 90 \\
9 & 82 & 83 & 85 & 88 & 73 & 88 & \\
Média & & 99,44 & $67,00^{\mathrm{a}}$ & $83,22^{\mathrm{a}}$ & 96,22 & $84,17^{\mathrm{a}}$ & $87,33^{\mathrm{b}}$ \\
Desvio padrão & 18,34 & 11,31 & 8,20 & 17,68 & 8,68 & 3,06
\end{tabular}

${ }^{a}$ Valor estatisticamente significante em relação a T0.

${ }^{\mathrm{b}}$ Valor não estatisticamente significante em relação a T0.

Quadro 3. Valores da $\mathrm{PaO}_{2}$ (mmHg) obtidos nos diferentes tempos de avaliação e momentos nos animais submetidos à sutura mecânica com grampeador cirúrgico (Grupo B)

\begin{tabular}{|c|c|c|c|c|c|c|c|}
\hline Subgrupo & Cão & T0 & T1EXT & $\mathrm{T} 48 \mathrm{~h}$ & T7d & $\mathrm{T} 15 \mathrm{~d}$ & T36d \\
\hline & 10 & 74 & 79 & 93 & 83 & - & - \\
\hline \multirow[t]{3}{*}{ B1 } & 11 & 81 & 82 & 89 & 90 & - & - \\
\hline & 12 & 83 & 90 & 85 & 77 & - & - \\
\hline & 13 & 93 & 100 & 74 & 69 & 76 & - \\
\hline \multirow[t]{3}{*}{ B2 } & 14 & 88 & 91 & 70 & 83 & 93 & - \\
\hline & 15 & 81 & 72 & 75 & 80 & 83 & - \\
\hline & 16 & 94 & 88 & 83 & 92 & 91 & 98 \\
\hline \multirow[t]{2}{*}{ B3 } & 17 & 95 & 87 & 90 & 97 & 91 & 93 \\
\hline & 18 & 94 & 94 & 82 & 92 & 84 & 92 \\
\hline Média & & 87,00 & 87,00 & 82,33 & 84,78 & 88,00 & 94,33 \\
\hline Desvio padrão & & 7,55 & 8,35 & 7,91 & 8,80 & 7,04 & 3,21 \\
\hline
\end{tabular}

Quadro 4. Valores da $\mathrm{PaCO}_{2}$ (mmHg) obtidos nos diferentes tempos de avaliação e momentos nos animais submetidos à sutura manual do coto brônquico (Grupo A)

\begin{tabular}{cccccccc}
\hline Subgrupo & Cão & T0 & T1EXT & T48h & T7d & T15d & T36d \\
\hline \multirow{4}{*}{ A1 } & 1 & 27 & 33 & 33 & 33 & - & - \\
& 2 & 29 & 39 & 36 & 34 & - & - \\
& 3 & 27 & 29 & 27 & 28 & - & - \\
A2 & 4 & 28 & 34 & 30 & 36 & 28 & - \\
& 5 & 30 & 29 & 26 & 37 & 30 & - \\
& 6 & 34 & 35 & 34 & 33 & 35 & - \\
A3 & 7 & 35 & 37 & 33 & 35 & 36 & 38 \\
& 8 & 32 & 29 & 31 & 33 & 29 & 31 \\
Média & 9 & 36 & 35 & 37 & 33 & 35 & 37 \\
Desvio padrão & & 30,89 & 33,33 & 31,89 & 33,56 & 32,17 & 35,33 \\
& & 3,48 & 3,67 & 3,76 & 2,55 & 3,54 & 3,79
\end{tabular}

considerados normais para a espécie. De outro modo, os momentos T7d e T36d, cujos valores apesar de também terem apresentado valor inferior, não foram considerados estatisticamente significantes (Quadro 2).

Não houve diferenças significativas entre os valores da $\mathrm{PaO}_{2}$ antes e após a retirada do pulmão esquerdo nos animais do Grupo B. Apesar disto, algumas diferenças foram detectadas, como a diminuição da $\mathrm{PaO}_{2}$ em T48h e o aumento em T36d, quando comparado aos valores pré-operatórios, porém sem significado estatístico (Quadro 3).
$\mathrm{PaCO}_{2}$ (Tensão de dióxido de carbono arterial, $\mathrm{mmHg}$ )

Observou-se que não houve diferenças significativas entre os valores da $\mathrm{PaCO}_{2}$ em T1EXT, T48h, T7d, T15d e T36d em comparação com os valores do T0, para os subgrupos A1, A2 e A3 (Quadro 4).

Estatisticamente não foram detectadas diferenças significativas entre a $\mathrm{PaCO}_{2}$ nos diferentes momentos avaliados para os Subgrupos B1, B2 e B3 (Quadro 5).

\section{pH (Concentração do íon hidrogênio)}

Do mesmo modo, não foram encontradas diferenças significativas do pH em T1EXT, T48h, T7d, T15d e T36d com o

Quadro 5. Valores da $\mathrm{PaCO}_{2}$ (mmHg) obtidos nos diferentes tempos de avaliação e momentos nos animais submetidos à sutura mecânica com grampeador cirúrgico (Grupo B)

\begin{tabular}{cccccccc}
\hline Subgrupo & Cão & T0 & T1EXT & T48h & T7d & T15d & T36d \\
\hline \multirow{3}{*}{ B1 } & 10 & 30 & 35 & 27 & 37 & - & - \\
& 11 & 41 & 40 & 35 & 34 & - & - \\
& 12 & 33 & 33 & 31 & 42 & - & - \\
B2 & 13 & 30 & 27 & 29 & 31 & 35 & - \\
& 14 & 32 & 38 & 35 & 39 & 36 & - \\
& 15 & 36 & 42 & 32 & 35 & 32 & - \\
B3 & 16 & 32 & 33 & 41 & 33 & 32 & 34 \\
& 17 & 30 & 30 & 31 & 32 & 30 & 33 \\
Média & 18 & 29 & 33 & 33 & 34 & 36 & 31 \\
Desvio padrão & & 32,56 & 34,56 & 32,67 & 35,22 & 33,50 & 32,67 \\
& & 3,81 & 4,77 & 4,06 & 3,53 & 2,51 & 1,53
\end{tabular}

Quadro 6. Valores do $\mathrm{pH}$ obtidos nos diferentes tempos de avaliação e momentos nos animais submetidos à sutura manual do coto brônquico (Grupo A)

\begin{tabular}{cccccccc}
\hline Subgrupo & Cão & T0 & T1EXT & T48h & T7d & T15d & T36d \\
\hline \multirow{4}{*}{ A1 } & 1 & 7,47 & 7,41 & 7,42 & 7,43 & - & - \\
& 2 & 7,4 & 7,41 & 7,41 & 7,46 & - & - \\
& 3 & 7,44 & 7,43 & 7,44 & 7,39 & - & - \\
A2 & 4 & 7,42 & 7,38 & 7,43 & 7,4 & 7,42 & - \\
& 5 & 7,37 & 7,41 & 7,41 & 7,46 & 7,44 & - \\
& 6 & 7,39 & 7,39 & 7,43 & 7,42 & 7,43 & - \\
A3 & 7 & 7,47 & 7,4 & 7,43 & 7,43 & 7,4 & 7,4 \\
& 8 & 7,39 & 7,45 & 7,44 & 7,38 & 7,4 & 7,43 \\
Média & 9 & 7,4 & 7,38 & 7,4 & 7,38 & 7,44 & 7,38 \\
Desvio padrão & & 7,4167 & 7,4067 & 7,4233 & 7,4167 & 7,4217 & 7,403 \\
& 0,036 & 0,0229 & 0,0141 & 0,0312 & 0,0183 & 0,0251
\end{tabular}

Quadro 7. Valores de $\mathrm{pH}$ obtidos nos diferentes tempos de avaliação e momentos nos animais submetidos à sutura mecânica com grampeador cirúrgico (Grupo B)

\begin{tabular}{cccccccc}
\hline Subgrupo & Cão & T0 & T1EXT & T48h & T7d & T15d & T36d \\
\hline \multirow{3}{*}{ B1 } & 10 & 7,42 & 7,35 & 7,42 & 7,41 & - & - \\
& 11 & 7,42 & 7,42 & 7,41 & 7,4 & - & - \\
& 12 & 7,42 & 7,34 & 7,4 & 7,41 & - & - \\
B2 & 13 & 7,41 & 7,39 & 7,45 & 7,41 & 7,37 & - \\
& 14 & 7,4 & 7,33 & 7,4 & 7,4 & 7,42 & - \\
& 15 & 7,41 & 7,31 & 7,42 & 7,42 & 7,43 & - \\
B3 & 16 & 7,42 & 7,37 & 7,39 & 7,38 & 7,41 & 7,44 \\
& 17 & 7,42 & 7,4 & 7,41 & 7,42 & 7,41 & 7,4 \\
Média & 18 & 7,45 & 7,37 & 7,4 & 7,41 & 7,4 & 7,44 \\
Desvio padrão & & 7,4189 & 7,3644 & 7,4111 & 7,4067 & 7,4067 & 7,4270 \\
& 0,0136 & 0,0353 & 0,0176 & 0,0122 & 0,0206 & 0,0230
\end{tabular}


Quadro 8. Valores da $\left[\mathrm{HCO}_{3}^{-}\right](\mathrm{mEq} / \mathrm{L})$ obtidos nos diferentes tempos de avaliação e momentos nos animais submetidos à sutura manual do coto brônquico (Grupo A)

\begin{tabular}{cccccccc}
\hline Subgrupo & Cão & T0 & T1EXT & T48h & T7d & T15d & T36d \\
\hline \multirow{4}{*}{ A1 } & 1 & 19 & 21 & 20 & 21 & - & - \\
& 2 & 17 & 24 & 22 & 24 & - & - \\
& 3 & 17 & 19 & 18 & 16 & - & - \\
A2 & 4 & 17 & 19 & 19 & 21 & 17 & - \\
& 5 & 17 & 18 & 16 & 26 & 20 & - \\
& 6 & 20 & 20 & 22 & 21 & 23 & - \\
A3 & 7 & 24 & 23 & 21 & 22 & 22 & 23 \\
& 8 & 19 & 20 & 21 & 19 & 17 & 20 \\
Média & 9 & 21 & 20 & 21 & 18 & 23 & 21 \\
Desvio padrão & & 19,00 & 20,44 & 20,00 & 20,89 & 20,33 & 21,33 \\
& 2,39 & 1,94 & 2,00 & 3,02 & 2,80 & 1,53
\end{tabular}

Quadro 9. Valores da $\left[\mathrm{HCO}_{3}^{-}\right]$(mEq/L) obtidos nos diferentes tempos de avaliação e momentos nos animais submetidos à sutura mecânica com grampeador cirúrgico (Grupo B)

\begin{tabular}{cccccccc}
\hline Subgrupo & Cão & T0 & T1EXT $^{\mathrm{a}}$ & T48h & T7d & T15d & T36d \\
\hline \multirow{3}{*}{ B1 } & 10 & 19 & 19 & 17 & 23 & - & - \\
& 11 & 26 & 25 & 21 & 20 & - & - \\
& 12 & 21 & 18 & 18 & 26 & - & - \\
B2 & 13 & 18 & 16 & 20 & 19 & 19 & - \\
& 14 & 19 & 19 & 21 & 24 & 23 & - \\
& 15 & 22 & 21 & 20 & 20 & 19 & - \\
B3 & 16 & 19 & 19 & 24 & 18 & 20 & 22 \\
& 17 & 19 & 18 & 18 & 20 & 18 & 20 \\
Média & 18 & 20 & 18 & 20 & 21 & 21 & 20 \\
Desvio padrão & & 20,33 & 19,22 & 19,89 & 21,22 & 20,00 & 20,67 \\
& 2,45 & 2,54 & 2,09 & 2,59 & 1,79 & 1,15
\end{tabular}

a Valor estatisticamente significante em relação a T0.

Quadro 10. Valores da frequiência cardíaca (bpm) obtidos nos diferentes tempos de avaliação e momentos nos animais submetidos à sutura manual do coto brônquico (Grupo A)

\begin{tabular}{cccccccc}
\hline Subgrupo & Cão & T0 & T1EXT & T48h & T7d & T15d & T36d \\
\hline \multirow{4}{*}{ A1 } & 1 & 162 & 141 & 108 & 118 & - & - \\
& 2 & 160 & 120 & 120 & 96 & - & - \\
& 3 & 80 & 104 & 95 & 80 & - & - \\
A2 & 4 & 130 & 92 & 120 & 140 & 148 & - \\
& 5 & 96 & 112 & 100 & 100 & 84 & - \\
& 6 & 93 & 92 & 120 & 123 & 129 & - \\
A3 & 7 & 118 & 80 & 105 & 130 & 144 & 108 \\
& 8 & 113 & 124 & 132 & 104 & 112 & 137 \\
Média & 9 & 114 & 82 & 114 & 120 & 140 & 140 \\
Desvio padrão & & 20,33 & 19,22 & 19,89 & 21,22 & 20,00 & 20,67 \\
& 2,45 & 2,54 & 2,09 & 2,59 & 1,79 & 1,15
\end{tabular}

controle pré-operatório para os Subgrupos A1, A2 e A3 (Quadro 6).

Com relação ao pH para o Grupo B, diferenças significativas ocorreram entre o T0 e o T1EXT $(p=0,011)$. No T1EXT, os Cães $10,12,14,15,16$ e 18 apresentaram valores abaixo da normalidade. Apesar de tal alteração, estes animais não desenvolveram acidose, segundo os valores padrões estipulados para cada variável (Quadro 7).

\section{[HCO3] (Concentração do íon bicarbonato, mEq/L)}

Em relação à avaliação pré-operatória, não houve diferen-
Quadro 11. Valores da frequiência cardíaca (bpm) obtidos nos diferentes tempos de avaliação e momentos nos animais submetidos à sutura mecânica com grampeador cirúrgico (Grupo B)

\begin{tabular}{cccccccc}
\hline Subgrupo & Cão & T0 & T1EXT & T48h & T7d & T15d & T36d \\
\hline \multirow{3}{*}{ B1 } & 10 & 180 & 82 & 93 & 100 & - & - \\
& 11 & 160 & 118 & 121 & 224 & - & - \\
& 12 & 116 & 91 & 122 & 128 & - & - \\
B2 & 13 & 116 & 100 & 108 & 112 & 172 & - \\
& 14 & 115 & 93 & 148 & 104 & 123 & - \\
& 15 & 115 & 83 & 99 & 120 & 91 & - \\
B3 & 16 & 120 & 97 & 143 & 115 & 125 & 127 \\
& 17 & 106 & 112 & 123 & 108 & 83 & 84 \\
Média & 18 & 115 & 89 & 96 & 124 & 111 & 103 \\
Desvio padrão & & 127 & 96,11 & 117 & 126,11 & 117,5 & 104,66 \\
& 25,15 & 12,27 & 19,79 & 37,83 & 31,59 & 21,54
\end{tabular}

Quadro 12. Valores da frequiência respiratória ( $\mathrm{mpm}$ ) obtidos nos diferentes tempos de avaliação e momentos nos animais submetidos à sutura manual do coto brônquico (Grupo A)

\begin{tabular}{cccccccc}
\hline Subgrupo & Cão & T0 & T1EXT & T48h & T7d & T15d & T36d \\
\hline \multirow{3}{*}{ A1 } & 1 & 56 & 35 & 68 & 80 & - & - \\
& 2 & 108 & 48 & 104 & 44 & - & - \\
& 3 & 32 & 34 & 40 & 21 & - & - \\
A2 & 4 & 41 & 48 & 28 & 27 & 22 & - \\
& 5 & 25 & 30 & 25 & 25 & 33 & - \\
& 6 & 22 & 28 & 20 & 20 & 31 & - \\
A3 & 7 & 44 & 31 & 35 & 35 & 40 & 32 \\
& 8 & 27 & 32 & 24 & 32 & 32 & 39 \\
Média & 9 & 32 & 24 & 25 & 37 & 48 & 42 \\
Desvio padrão & & 43 & 33,33 & 41 & 35,66 & 34,33 & 37,66 \\
& & 26,60 & 9,44 & 27,71 & 18,37 & 8,82 & 5,13
\end{tabular}

Quadro 13. Valores da frequiência respiratória $(\mathrm{mpm})$ obtidos nos diferentes tempos de avaliação e momentos nos animais submetidos à sutura mecânica com grampeador cirúrgico (Grupo B)

\begin{tabular}{cccccccc}
\hline Subgrupo & Cão & T0 & T1EXT & T48h & T7d & T15d & T36d \\
\hline \multirow{2}{*}{ B1 } & 10 & 83 & 25 & 48 & 57 & - & - \\
& 11 & 29 & 22 & 23 & 32 & - & - \\
& 12 & 32 & 21 & 28 & 24 & - & - \\
B2 & 13 & 84 & 33 & 78 & 87 & 88 & - \\
& 14 & 41 & 38 & 68 & 95 & 78 & - \\
& 15 & 64 & 14 & 35 & 31 & 33 & - \\
B3 & 16 & 60 & 38 & 39 & 41 & 40 & 41 \\
& 17 & 46 & 40 & 33 & 37 & 35 & 28 \\
Média & 18 & 56 & 34 & 32 & 31 & 33 & 41 \\
Desvio padrão & 20,04 & 9,19 & 18,70 & 25,95 & 25,95 & 24,99 & 7,50
\end{tabular}

ças significativas dos valores da $\left[\mathrm{HCO}_{3}^{-}\right]$em T1EXT, T48h, T7d, T15d e T36d para os subgrupos A1, A2 e A3; apenas o Cão 5 do Subgrupo A2 apresentou no T7d valor acima do padrão da normalidade para a espécie, porém estatisticamente não significante (Quadro 8).

Já em relação ao T0, houve alteração significativa, quando comparada com o T1EXT $(p=0,26)$. Entretanto, a maioria dos valores referentes à $\left[\mathrm{HCO}_{3}^{-}\right]$para os animais dos Subgrupos B1, B2 e B3 se mantiveram dentro do intervalo fisiológico (Quadro 9). 
De acordo com os valores obtidos referentes à frequiência cardíaca (batimentos por minuto, bmp) e frequiência respiratória (movimentos por minuto, $\mathrm{mpm}$ ) não houve diferença significativa entre os Grupos A e B (Quadros 10 a 13).

\section{DISCUSSÃO}

Os valores obtidos nos animais de ambos os grupos no período pré-operatório, quanto aos parâmetros $\mathrm{PaO}_{2}, \mathrm{PaCO}_{2}, \mathrm{pH}$ e $\left[\mathrm{HCO}_{3}{ }^{-}\right]$estavam, em sua maioria, dentro do intervalo da normalidade padronizado para o sangue arterial segundo Massone (1999) e DiBartola (2006). Aqueles que apresentaram algum valor acima ou abaixo do limite fisiológico não foram retirados do experimento, pois estavam em condições clínicas julgadas satisfatórias com base em exame físico, laboratorial (hematológico e bioquímico), radiográfico e broncoscópico, além de não se encontrarem em significativo desequilíbrio ácido-básico ou com hipoxemia importante.

Após a pneumonectomia, houve redução significativa da $\mathrm{PaO}_{2}$, observada no Grupo A no T1EXT dos Subgrupos A1, A2 e A3. Entretanto, verificou-se que, nos momentos seguintes, a $\mathrm{PaO}_{2}$ já retornava a valores do intervalo fisiológico para, no T36d, não mais apresentar diferenças significativas em relação ao T0. Estes resultados corroboram com os obtidos por Carlin et al. (1991), Hsia et al. (1994) e Takeda et al. (1999) no pós-operatório mediato. Esta hipoxemia momentânea verificada uma hora após a extubação pode estar relacionada a diferentes fatores. A técnica de sutura manual envolve maior manipulação e tempo cirúrgico, por conseguinte, maior chance de atelectasia do pulmão a despeito da manobra de recrutamento alveolar realizada ao término da anestesia em ambos os grupos. Outro motivo aventado seria o efeito residual da anestesia geral e dos fármacos analgésicos empregados ao término da intervenção operatória. Neste aspecto, entretanto, deve-se ressaltar que a frequiência respiratória não diminuiu de forma significativa no pós-operatório imediato, o que poderia estar associado à excessiva sedação ou depressão promovida pelos anestésicos e analgésicos utilizados. Fato que vem corroborar essa observação são os valores da $\mathrm{PaCO}_{2}$ verificados no grupo $\mathrm{A}$, nesse mesmo momento, que não denotam nenhum grau de depressão respiratória. Alterações da frequiência cardíaca também poderiam estar relacionadas com dor e esta, por conseguinte, ocasionaria dificuldade respiratória. Nenhuma alteração significativa neste parâmetro foi verificada ao longo do período de observação. Isto posto, nos parece que o fator contribuinte principal para a ocorrência de hipoxemia no grupo de animais submetidos a sutura manual do coto brônquico, tenha sido de fato a ocorrência de atelectasia por maior manipulação do pulmão. Durante a anestesia, procurou-se empregar uma $\mathrm{FIO}_{2}$ de $80 \%$ pois quando se emprega $100 \%$ de oxigênio, a chance de atelectasia pósoperatória é muito maior (Galiatsou et al. 2006).

Durante a avaliação de todos os momentos pós-operatórios, foi mantido o equilíbrio ácido-básico, vale dizer que nenhum animal dos Grupos A e B desenvolveu alcalose ou acidose após a exérese do pulmão esquerdo, à semelhança ao observado por Wilcox et al. (1979), Hsia et al. (1990), Hjaimarsen et al. (1995) e Miyazawa et al. (1999) em seus estudos.
Diferente do Grupo A, os cães do Grupo B não apresentaram redução significativa da $\mathrm{PaO}_{2}$ imediatamente após a pneumonectomia esquerda, no T1EXT, quando comparados com a avaliação pré-operatória, permanecendo sempre dentro do intervalo fisiológico para a espécie canina. Estes resultados vêm ao encontro daqueles descritos por Carlin et al. (1991), Hsia et al. (1994) e Takeda et al. (1999) no pós-operatório mediato, como já ressaltado no segundo parágrafo da discussão.

Alguns autores abordam assuntos próximos ao proposto nesta pesquisa. Merecem destaque Matsumoto et al. (2004), que desenvolveram um estudo no qual cães foram submetidos a pneumonectomia esquerda seguida de implantação autóloga do lobo caudal esquerdo, sendo que não houve diferença significativa nos valores da $\mathrm{PaO}_{2}$ verificados antes e após a intervenção cirúrgica. Posteriormente, Nagao \& Cataneo (2005) realizaram estudo semelhante e verificaram que não houve diferença significativa dos valores gasométricos nos diferentes momentos cirúrgicos, ficando os valores médios em $\mathrm{pH}=7,37, \mathrm{PaO}_{2}=87 \mathrm{mmHg}$ e $\mathrm{PaCO}_{2}=53 \mathrm{mmHg}$.

Na revisão da literatura, poucos foram os trabalhos que avaliaram valores hemogasométricos na intervenção cirúrgica de pneumonectomia em cães, como Hsia et al. (2003), que corroboram os dados ora observados, impossibilitando a elaboração de discussão mais ampla. Apesar disso, pode-se afirmar, certamente, que todos os dados logrados em nossa pesquisa serão de grande valia para a avaliação da viabilidade de aplicação em rotina de tal intervenção cirúrgica na medicina veterinária, principalmente quanto à preservação da função pulmonar após a excisão do pulmão esquerdo.

\section{CONCLUSÕES}

Os cães submetidos à pneumonectomia esquerda (com sutura manual ou mecânica do coto brônquico esquerdo) não tendem a apresentar desequilíbrio ácido-básico imediato e mediato até 36 dias de pós-operatório.

A oxigenação sanguínea arterial apresenta-se reduzida no pós-operatório imediato dos animais pneumonectomizados submetidos à sutura manual, com rápida tendência de retorno aos valores fisiológicos para a espécie canina.

Agradecimentos.- À FAPESP pelo apoio financeiro que proporcionou a realização deste trabalho (Proc.00/08578-1).

\section{REFERÊNCIAS}

Carlin J.I., Hsia C.C.W., Cassidy S.S., Ramanathan M., Clifford P.S. \& Johnson R.L.J. 1991. Recruitment of lung diffusing capacity with exercise before and after pneumonectomy in dogs. J. Appl. Physiol. 70:135-142.

DiBartola S.P. 2006. Fluid, Electrolyte, and Acid-Base Disorders in Small Animal Practice. $3^{\text {rd }}$ ed. Saunders Elsevier, St Louis, Missouri, p.229-250.

Galiatsou E., Kostanti E., Svarna E., Kitsakos A., Koulouras V., Efremidis S.C. \& Nakos G. 2006. Prone position augments recruitment and prevents alveolar overinflation in acute lung injury. Am. J. Respiratory and Critical Care Medicine 174(2):187-97.

Goire J., Deslauriers J., Ghojin L. \& Rouleau J. 1993. Indications, risks, and results of completion pneumonectomy. J. Thoracic and Cardiovascular Surgery 105:918-24.

Hawkins E.C. 2006. Manifestações clínicas dos distúrbios do trato respirató- 
rio inferior, p.241-245. In: Couto C.G. \& Nelson W.N. (ed.), Medicina Interna de Pequenos Animais. $3^{\underline{a}}$ ed. Elsevier, São Paulo.

Hjalmarsen A., Aasebo U. \& Lie M. 1995. Pulmonary function 3-12 months after pneumonectomy, lobectomy or bilobectomy for lung cancer. Scand. J. Thoracic Cardiovasc. Surg. 29:71-74.

Hsia C.C.W., Carlin J.I., Wagner P.D., Cassidy S.S. \& Johnson R.L.J. 1990. Gas exchange abnormalities after pneumonectomy in conditioned foxhounds. J. Appl. Physiol. 68:94-104.

Hsia C.C.W., Herazo L.F., Fryder-Doffey F. \& Weibel E.R. 1994. Compensatory lung growth occurs in adult dogs after right pneumonectomy. J. Clin. Invest. 94:405-412.

Hsia C.C.W., Johnson R.L.J., Wu E.Y, Estrera A.S., Wagner H. \& Wagner P.D. 2003. Reducing lung strain after pneumonectomy impairs oxygen diffusing capacity but not ventilitation-perfusion matching. J. Appl. Physiol. 95:13701378.

Irino E.T. 2006. Avaliação histopatológica do pulmão esquerdo e avaliação eletrocardiográfica em cães (Canis familiaris) submetidos à pneumonectomia direita. Dissertação de Mestrado, Departamento de Cirurgia, Faculdade de Medicina Veterinária e Zootecnia, Universidade de São Paulo. 85p.

Irino E.T., Stopiglia A.J., Larsson M.H.M.A, Guerra J.L., Simões E.A., Shinkai M.T., Aya D.O., Saldiva P.H.N. \& Jatene F.B. 2004. Avaliação eletrocardiográfica e avaliação histopatológica do pulmão direito em cães $(\mathrm{Ca}-$ nis familiaris) submetidos à pneumonectomia esquerda. Braz. J. Vet. Res. Anim. Sci. 41(1):58-66.

Massone F. 1999. Apêndices, p.196-213. In: Massone F. (ed.), Anestesiologia Veterinária. $3^{\underline{a}}$ ed. Guanabara Koogan, Rio de Janeiro.

Matsumoto I., Oda M., Tsunezuka Y., Tamura M., Kawakami K. \& Watanabe G. 2004. Experimental study of extracorporeal lung resection in dogs: EX situ sleeve resection and autotransplantation of the pulmonary lobe after extended pneumonectomy for central lung cancer. J. Thoracic Cardiovasc. Surg. 127:1343-1349.

Miyazawa M., Haniuda M., Nishimura H. \& Kubo K. 1999. Long-term effects of pulmonary resection on cardiopulmonary function.J. Am. Coll. Surgeons 189:26-33.

Nagao R.K. \& Cataneo A.J.M. 2005. Implante lobar autólogo póspneumonectomia: modelo experimental em cães. Acta Cirúrg. Bras. 20(4):311-316

Simões E.A. 2003. Estudo comparativo entre sutura mecânica e manual em brônquio após pneumonectomia esquerda em cães: uma avaliação anátomo-patológica, paramétrica, radiológica e broncoscópica. Dissertação de Mestrado, Departamento de Cirurgia, Faculdade de Medicina Veterinária e Zootecnia, USP, São Paulo. 107p.

Simões E.A., Jatene F.B., Stopiglia A.J., Fantoni D.T., Guerra J.L., Aielo V.D., Irino E.T., Aya D.O., Binoki D.H., Freitas R.R. \& Monteiro R. 2005. Estudo comparativo entre sutura mecânica e manual em brônquio após pneumonectomia esquerda em cães (Canis familiaris): uma avaliação anatomopatológica, paramétrica, radiológica e broncoscópica. Braz. J. Vet. Res. Anim. Sci. 42(6):357-366

Takeda S.I., Hsia C.C.W., Wagner E, Ramanathan M., Estrera A.S. \& Weibel E.R. 1999. Compensatory alveolar growth normalizes gas-exchange function in immature dogs after pneumonectomy. J. Appl. Physiol. 86:1301-1310.

Thompson S.E. \& Johnson, J.M. 1991. Analgesia in dogs after intercostals thoracotomy: a comparison of morfine, selective intercostal nerve block and interpleural regional analgesia with bupivacaine. Vet. Surg. 20(1):7377 .

Wilcox B.R., Murray G.F., Friedman M. \& Pimmel R.L. 1979. The effects of early pneumonectomy on the remaining pulmonary parenchyma. Surgery 86:294-300. 